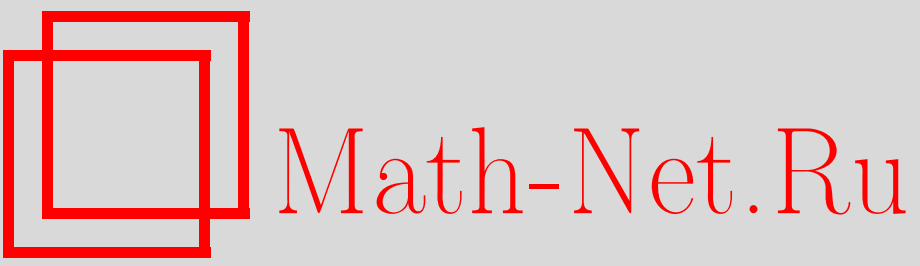

В. А. Смирнов, Четырехмерное интегрирование по частям с дифференциальной перенормировкой как метод вычисления диаграмм Фейнмана, ТМФ, 1996 , том 108, номер 1, 129-134

DOI: https://doi.org/10.4213/tmf1182

Использование Общероссийского математического портала Math-Net.Ru подразумевает, что вы прочитали и согласны с пользовательским соглашением

http: //www . mathnet.ru/rus/agreement

Параметры загрузки:

IP : 54.80 .73 .141

26 апреля 2023 г., 18:18:11 


\section{ЧЕТЫРЕХМЕРНОЕ ИНТЕГРИРОВАНИЕ ПО ЧАСТЯМ С ДИФФЕРЕНЦИАЛЬНОЙ ПЕРЕНОРМИРОВКОЙ КАК МЕТОД ВЫЧИСЛЕНИЯ ДИАГРАММ ФЕЙНМАНА}

Показано, как четырехмерное интегрирование по частям в сочетании с дифференциальной перенормировкой и ее инфракрасным аналогом может применяться для вычисления диаграмм Фейнмана.

1. Интегрирование по частям [1] в рамках размерной регуляризации [2] оказалось одним из наиболее мощных методов выгисления диаграмм Фейнмана. Несмотря на то что размерная регуляризация и перенормировка [3] обычно используются в практических вычислениях, они оказываются неудобными в ситуациях, связанных с киральной и суперсимметриями. Цель настоящей краткой заметки - предложить четырехмерную версию этого метода вычисления. Будет показано, как (обычное) четырехмерное интегрирование по частям в совокупности с техникой дифференциальной перенормировки [4-9] и ее инфракрасного (ИК) аналога ( $\widetilde{R}$-операции) может быть использовано для вычисления диаграмм.

В следуюшем пункте будут описаны предписания дифференциальной перенормировки и $\widetilde{R}$-операции для простейших диаграмм. С использованием примера базисной двухпетлевой диаграммы в пункте 3 будет показано, как четырехмерное интегрирование по частям применяется в вычислениях. В заключение обсуждаются перспективы применения предложенного метода.

2. Дифференциальная перенормировка впервые была определена в координатном пространстве [4-8]. Можно перевести ее рецепты на язык импульсного пространства. Другой вариант - использовать однородные свойства фейнмановских интегралов и сформулировать соответствующий рецепт в импульсном пространстве - cм. [9], где это предписание было сформулировано для логарифмически расходяшихся диаграмм с простейшей топологией подрасходимостей. (Возможно, более естественно называть эту перенормировку однородной, а не дифференциальной.) В частности, для простейшего однопетлевого фейнмановского интеграла ${ }^{1)}$ пропагаторного типа имеем следуюшее перенормировочное предписание [9]:

$$
R F(q) \equiv R \int d^{4} k \frac{1}{k^{2}(q-k)^{2}}=\int d^{4} k \ln \frac{k^{2}}{\mu^{2}} \cdot \frac{1}{2} q \frac{\partial}{\partial q} \frac{1}{k^{2}(q-k)^{2}} .
$$

\footnotetext{
1) Для простоты рассматриваются фейнмановские интегралы в евклидовом пространстве.
} 
Здесь $\mu$ - массивный параметр, который учитывает конечный произвол перенормировки. Производная по $q$ применяется перед интегрированием, так что соответствуюший интеграл оказывается сходящимся.

Чтобы вычислить (1), разумно ввести аналитическую регуляризацию [10]

$$
R F(q)=\left.\int d^{4} k\left(-\frac{d}{d \lambda}\right) \frac{1}{2} q \frac{\partial}{\partial q} \frac{\mu^{2 \lambda}}{\left(k^{2}\right)^{1+\lambda}(q-k)^{2}}\right|_{\lambda=0} .
$$

Когда $\lambda \neq 0$, можно использовать следуюший порядок: вычислить интеграл, продифференцировать по $q$, затем по $\lambda$ и, наконец, положить $\lambda=0$. При вычислении интеграла используется четырехмерная формула однопетлевого интегрирования

$$
\int d^{4} k \frac{1}{\left(k^{2}\right)^{\lambda_{1}}\left((q-k)^{2}\right)^{\lambda_{2}}}=\pi^{2} G\left(\lambda_{1}, \lambda_{2}\right) \frac{1}{\left(q^{2}\right)^{\lambda_{1}+\lambda_{2}-2}},
$$

где $G$ - четырехмерная $G$-функция

$$
G\left(\lambda_{1}, \lambda_{2}\right)=\frac{\Gamma\left(\lambda_{1}+\lambda_{2}-2\right)}{\Gamma\left(\lambda_{1}\right) \Gamma\left(\lambda_{2}\right)} \frac{\Gamma\left(2-\lambda_{1}\right) \Gamma\left(2-\lambda_{2}\right)}{\Gamma\left(4-\lambda_{1}-\lambda_{2}\right)} .
$$

В частности,

$$
G(1,1+\lambda)=\frac{1}{\lambda(1-\lambda)}
$$

В результате имеем

$$
R \int d^{4} k \frac{1}{k^{2}(q-k)^{2}}=\pi^{2}\left(1-\ln q^{2} / \mu^{2}\right) .
$$

Нам понадобится также ИК-аналог дифференциальной перенормировки. В случае размерной перенормировки соответствуюший ИК-аналог был описан в работах [11]. Эта операция $\widetilde{R}$ удаляет ИК-расходимости (вне массовой оболочки) вполне аналогично тому, как обычная размерная перенормировка удаляет ультрафиолетовые (УФ) расходимости. Когда она объединяется с само́й $R$-операцией, получается так называемая $R^{*}$-операция [11] $\left(R^{*}=\widetilde{R} R\right)$, которая успешно применяется в ренормгрупповых вычислениях и асимптотических разложениях фейнмановских интегралов в различных пределах импульсов и масс (см., например, обзор [12]).

Дифференциальную $\widetilde{R}$-операцию в импульсном пространстве можно определить аналогично тому, как дифференциальная перенормировка определяется в координатном пространстве ${ }^{2)}$. Для простейшего ИК-расходяшегося подграфа, состоящего из двух последовательных безмассовых скалярных линий, которые порождают неинтегрируемый множитель $1 / k^{4}$, имеем следующее предписание:

$$
\widetilde{R} \frac{1}{k^{4}}=\frac{1}{2} \frac{\partial}{\partial k} k\left(\frac{\ln k^{2} / \widetilde{\mu}^{2}}{k^{4}}\right)
$$

\footnotetext{
2) Точнее говоря, УФ-перенормировочные предписания в координатном пространстве, сфформулированные в работах $[6,8]$, переформулируются в виде "ИК-перенормировочных" предписаний в импульсном пространстве с использованием УФ-ИК-аналогии (как это делалось в случае размерной $\widetilde{R}$-операции, см. [11, 12]). Отметим, что в работе [13] авторы сделали вывод о противоречивости $\widetilde{R}$-операции на основании того, что они (в рамках своей версии $\widetilde{R}$-операции) получили разные результаты, интегрируя в разных порядках. Этот вывод представляется неправомерным, так как у авторов не был сформулирован общий рецепт $\widetilde{R}$-операции (для произвольной диаграммы), а для конкретных рассмотренных диаграмм разные ответы получились за счет того, что разный порядок интегрирования фактически соответствовал разным ИК-перенормировочным предписаниям.
} 
где $\widetilde{\mu}$ - ИК-перенормировочный параметр. Отсюда для однопетлевого ИК-расходящегося фейнмановского интеграла

$$
\int d^{4} k \frac{1}{k^{4}(q-k)^{2}}
$$

получаем следующее “ $\widetilde{R}$-нормированное” выражение:

$$
\widetilde{R} \int d^{4} k \frac{1}{k^{4}(q-k)^{2}}=\int d^{4} k\left(\frac{1}{2} \frac{\partial}{\partial k} k \frac{\ln k^{2} / \widetilde{\mu}^{2}}{k^{4}}\right) \frac{1}{(q-k)^{2}} .
$$

Производная в формулах (7) и (8) понимается в смысле теории обобщенных функций (что формально эквивалентно четырехмерному интегрированию по частям), т.е.

$$
\widetilde{R} \int d^{4} k \frac{1}{k^{4}(q-k)^{2}}=\int d^{4} k \frac{k(k-q) \ln k^{2} / \widetilde{\mu}^{2}}{k^{4}(q-k)^{4}} .
$$

Чтобы вычислить интеграл в (9), полезно ввести аналитическую регуляризацию, а затем продифференцировать подынтегральное выражение при $\lambda \neq 0$, используя соотношение $\frac{1}{2} \frac{\partial}{\partial k} k \frac{\tilde{\mu}^{2 \lambda}}{\left(k^{2}\right)^{2+\lambda}}=-\lambda \frac{\widetilde{\mu}^{2 \lambda}}{\left(k^{2}\right)^{2+\lambda}}$. Получим

$$
\widetilde{R} \int d^{4} k \frac{1}{k^{4}(q-k)^{2}}=\pi^{2} \frac{1}{q^{2}}\left(1+\ln q^{2} / \widetilde{\mu}^{2}\right) .
$$

Для последуюших вычислений будут нужны также похожие интегралы с дополнительными логарифмами, которые тоже вычисляются с помошью аналитической регуляризациии формулы однопетлевого интегрирования (3). Получаем

$$
\begin{gathered}
\widetilde{R} \int d^{4} k \frac{\ln \left((q-k)^{2} / \mu^{2}\right)}{k^{4}(q-k)^{2}}=\int d^{4} k\left(\frac{1}{2} \frac{\partial}{\partial k} k \frac{\ln k^{2} / \widetilde{\mu}^{2}}{k^{4}}\right) \frac{\ln (q-k)^{2} / \mu^{2}}{(q-k)^{2}}= \\
=\pi^{2} \frac{1}{q^{2}} \ln q^{2} / \mu^{2}\left(\ln q^{2} / \widetilde{\mu}^{2}+1\right) ; \\
\widetilde{R} \int d^{4} k \frac{\ln k^{2} / \widetilde{\mu}^{2}}{k^{4}(q-k)^{2}}=\int d^{4} k\left(\frac{1}{4} \frac{\partial}{\partial k} k \frac{\ln ^{2} k^{2} / \widetilde{\mu}^{2}}{k^{4}}\right) \frac{\ln (q-k)^{2} / \mu^{2}}{(q-k)^{2}}= \\
=\pi^{2} \frac{1}{q^{2}}\left(1+\ln q^{2} / \widetilde{\mu}^{2}+\frac{1}{2} \ln ^{2} q^{2} / \widetilde{\mu}^{2}\right) ; \\
\widetilde{R} \int d^{4} k \frac{\ln ^{2}\left((q-k)^{2} / \mu^{2}\right)}{k^{4}(q-k)^{2}}=\int d^{4} k\left(\frac{1}{2} \frac{\partial}{\partial k} k \frac{\ln k^{2} / \widetilde{\mu}^{2}}{k^{4}}\right) \frac{\ln ^{2}(q-k)^{2} / \mu^{2}}{(q-k)^{2}}= \\
=\pi^{2} \frac{1}{q^{2}}\left(\ln ^{2} q^{2} / \mu^{2}\left(\ln q^{2} / \widetilde{\mu}^{2}+1\right)+4 \zeta(3)\right) ; \\
\widetilde{R} \int d^{4} k \frac{\ln \left((q-k)^{2} / \mu^{2}\right) \ln k^{2} / \widetilde{\mu}^{2}}{k^{4}(q-k)^{2}}=\int d^{4} k\left(\frac{1}{4} \frac{\partial}{\partial k} k \frac{\ln ^{2} k^{2} / \widetilde{\mu}^{2}}{k^{4}}\right) \frac{\ln (q-k)^{2} / \mu^{2}}{(q-k)^{2}}= \\
=\pi^{2} \frac{1}{q^{2}}\left(\ln q^{2} / \mu^{2}\left(1+\ln q^{2} / \widetilde{\mu}^{2}+\frac{1}{2} \ln ^{2} q^{2} / \widetilde{\mu}^{2}\right)+2 \zeta(3)\right),
\end{gathered}
$$




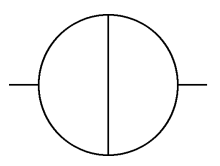

Базисная двухпетлевая диаграмма

где $\zeta(z)$ - дзета-функция Римана.

3. Чтобы вычислить базисную двухпетлевую диаграмму (см. рисунок) в точности в четырех измерениях

$$
J(q)=\iint \frac{d^{4} k d^{4} l}{k^{2}(k-q)^{2}(k-l)^{2} l^{2}(l-q)^{2}},
$$

применим четырехмерное интегрирование по частям

$$
\iint d^{4} k d^{4} l \frac{\partial}{\partial l_{\alpha}}\left\{\frac{(k-l)_{\alpha}}{k^{2}(k-q)^{2}(k-l)^{2} l^{2}(l-q)^{2}}\right\}=0 .
$$

Формально это то же самое тождество, которое использовалось в рамках стандартного метода интегрирования по частям [1], основанного на размерной регуляризации. Используя соотношения $2(k-l) l=k^{2}-(k-l)^{2}-l^{2}, 2(k-l)(l-q)=(k-q)^{2}-(k-l)^{2}-(l-q)^{2}$, получаем

$$
\iint d^{4} k d^{4} l \frac{(k-l)^{2}-(k-q)^{2}}{k^{2}(k-q)^{2}(k-l)^{2} l^{2}(l-q)^{4}}=0 .
$$

Хотя этот интеграл УФ- и ИК-сходящийся, расходимости обоих типов получаются при отдельном рассмотрении двух слагаемых в числителе. Можно, однако, отделить их, вводя (заранее) $R$ - и $\widetilde{R}$-операции. Например, для $R$-операции используются следующие тождества (при $j=1,2, \ldots)$ :

$$
\int d k \ln ^{j} l^{2} / \widetilde{\mu}^{2} \Pi(k, l, q)=\int d k \ln k^{2} / \mu^{2} \ln ^{j} l^{2} / \tilde{\mu}^{2} \frac{1}{2}\left(q \frac{\partial}{\partial q}+l \frac{\partial}{\partial l}-\lambda-4\right) \Pi(k, l, q),
$$

где П - однородная функция переменных $k, l, q$ степени $\lambda$. После этого получаем

$$
R^{*} \iint d^{4} k d^{4} l \frac{1}{k^{2}(k-q)^{2} l^{2}(l-q)^{4}}=R^{*} \iint d^{4} k d^{4} l \frac{1}{k^{2}(k-l)^{2} l^{2}(l-q)^{4}},
$$

где $R^{*}=R \widetilde{R}$.

Применим теперь четырехмерное интегрирование по частям с дополнительным логарифмом

$$
\iint d^{4} k d^{4} l \frac{\partial}{\partial l_{\alpha}}\left\{\frac{(k-l)_{\alpha} \ln l^{2} / \widetilde{\mu}^{2}}{k^{2}(k-q)^{2}(k-l)^{2} l^{2}(l-q)^{2}}\right\}=0 .
$$

После этого приходим к соотношению

$$
\begin{aligned}
J(q)= & R \int d^{4} k \frac{1}{k^{2}(q-k)^{2}} \widetilde{R} \int d^{4} l \frac{\ln \left((q-l)^{2} / \widetilde{\mu}^{2}\right)}{l^{2}(q-l)^{4}}+ \\
& +R \int d^{4} k \frac{1}{k^{2}(q-k)^{2}} \widetilde{R} \int d^{4} l \frac{\ln l^{2} / \widetilde{\mu}^{2}}{l^{2}(q-l)^{4}}- \\
& -R^{*} \iint d^{4} k d^{4} l \frac{\ln l^{2} / \widetilde{\mu}^{2}}{k^{2}(k-l)^{2} l^{2}(q-l)^{4}}- \\
& -R^{*} \iint d^{4} k d^{4} l \frac{\ln \left((l-q)^{2} / \widetilde{\mu}^{2}\right)}{k^{2}(k-l)^{2} l^{2}(q-l)^{4}} .
\end{aligned}
$$


Для вычисления присутствующих рекурсивно-однопетлевых фейнмановских интегралов используются соотношения $(6),(10)-(14)$. В конце коншов получаем хорошо известный результат [14]:

$$
J(q)=6 \zeta(3) \pi^{4} / q^{2} .
$$

4. Как и в приведенном примере базисной двухпетлевой диаграммы, можно применять четырехмерное интегрирование по частям с дифференциальными $R$ - и $\widetilde{R}$-операциями по крайней мере в каждой ситуации, когда применяется интегрирование по частям в рамках размерной регуляризации. При этом надо использовать почти те же самые тождества [1], что и в случае размерной регуляризации. Грубо говоря, отрицательные степени параметра $\varepsilon=(4-d) / 2$ (где $d$ - размерность пространства-времени) заменяются на некоторые степени логарифмов от петлевых импульсов. Чтобы иметь возможность отдельно рассматривать слагаемые в этих тождествах, заранее удаляются искусственные УФ- и ИК-расходимости (в случае размерной регуляризации эти расходимости автоматически регуляризуются). Для проведения дальнейших вычислений нужна соответствуюшая таблиша однопетлевых интегралов с логарифмами (в рассмотренном случае это формулы (6), (10)-(14)).

Отметим, что в рамках аналитической регуляризации не удается использовать интегрирование по частям для вычисления базисной двухпетлевой диаграммы так, как это возможно в размерной регуляризации и в четырех измерениях в предложенной технике. Это объясняется невозможностью сокрашений типа $p^{2} / p^{2}=1$ в подынтегральных выражениях (из-за того, что в знаменателях появляется $p^{2}$ в степени, зависящей от параметра аналитической регуляризации). Поэтому, несмотря на то что таблица однопетлевых интегралов выведена с помощью аналитической регуляризации, появляющиеся логарифмы не мешают таким сокращениям (графически соответствующим стягиванию линий в точку), которые приводят к рекурсивно однопетлевым фейнмановским интегралам в правой части (21).

Этот факт вселяет оптимизм при построении дифференциальных перенормировочных схем, совместных с калибровочной инвариантностью, поскольку в конечном счете тождества Уорда связаны с возможностью упомянутых сокращений в перенормированных интегралах Фейнмана. Оказывается, действительно удается модифицировать предписания дифференциальной перенормировки работ $[6,8]$ так, что калибровочная инвариантность будет обеспечена автоматически (т.е. без "подгонки" конечных контрчленов) по крайней мере в абелевом случае ${ }^{3)}$. Этому будет посвящена отдельная работа.

В заключение подчеркнем, что для применений в теориях с киральной и суперсимметриями четырехмерная техника выглядит наиболее предпочтительной.

Я благодарен К.Г. Четыркину и Г.А. Кравцовой за ценные обсуждения.

Эта работа поддержана РФФИ, грант 96-01-00654.

\section{Список литературы}

[1] K.G.Chetyrkin, F.V.Tkachov // Nucl. Phys. 1981. V. B192. P. 159.

[2] G.'tHooft, M.Veltman // Nucl. Phys. 1972. V. B44. P. 189; C.G.Bollini, J.J.Giambiagi // Nuovo Cim. 1972. V. 12B. P. 20.

[3] P.Breitenlohner, D.Maison // Commun. Math. Phys. 1977. V. 52. P. 11, 39, 55.

\footnotetext{
${ }^{3)} \mathrm{Cм.} \mathrm{также} \mathrm{работу} \mathrm{[15],} \mathrm{где} \mathrm{в} \mathrm{рамках} \mathrm{дифференциальной} \mathrm{перенормировки} \mathrm{для} \mathrm{некоторых} \mathrm{спе-}$ циальных классов диаграмм доказаны соотношения, обеспечивающие тождества Уорда в КЭД.
} 
[4] D.Z.Freedman, K.Johnson, J.I.Latorre // Nucl. Phys. 1992. V. B371. P. 353.

[5] J.I.Latorre, C.Manuel, Cardona X.Vilasís // Ann. Phys. 1994. V. 231. P. 149.

[6] О.И.Завьялов, В.А.Смирнов // ТМФ. 1993. Т. 96. С. 288.

[7] V.A.Smirnov // Nucl. Phys. 1994. V. B427. P. 325.

[8] V.A.Smirnov // Z. Phys. C. 1995. V. 67. P. 531.

[9] О.И.Завьялов // ТМФ. 1994. Т. 98. С. 536.

[10] E.R.Speer // J. Math. Phys. 1968. V. 9. P. 1404.

[11] K.G.Chetyrkin, V.A.Smirnov // Phys. Lett. 1984. V. 144B. Р. 419; Препринт НИИЯФ МГУ 89-2/79. M., 1989.

[12] V.A.Smirnov. Renormalization and asymptotic expansions. Basel: Birkhäuser, 1991.

[13] L.V.Avdeev, D.I.Kazakov, I.N.Kondrashuk // Int. J. Mod. Phys. 1994. V. A9. P. 1067.

[14] J.L.Rosner // Ann. Phys. 1967. V. 44. P. 11.

[15] О.И.Завьялов, Г.А.Кравцова, А.М.Малокостов // ТМФ. 1996. Т. 107. № 1. С. 64-74.

НИИ ядерной физики МГУ

E-mail: smirnov@theory.npi.msu.su

Поступила в редакцию 27.IX.1996 г

\section{V.A. Smirnov \\ FOUR-DIMENSIONAL INTEGRATION BY PARTS WITH DIFFERENTIAL RENORMALIZATION AS A METHOD OF EVALUATION OF FEYNMAN DIAGRAMS}

It is shown how strictly four-dimensional integration by parts combined with differential renormalization and its infrared analogue can be applied for calculation of Feynman diagrams. 